\title{
Philosophie des mesures de radioprotection dans les centrales à eau pressurisée
}

\author{
J.-J. MARTIN * \\ (Manuscrit reçu le 30 juin 1978) \\ RÉSUMÉ
}

L'analyse des possibilités d'irradiation externe et de contamination atmosphérique existant dans une centrale PWR permet de mettre en évidence les moyens de mesure les mieux adaptés. Les mesures fixes apparaissent nécessaires dans certains cas, essentiellement en tant que détecteurs d'évolution, mais les mesures par appareils portatifs semblent plus appropriées pour l'évaluation des nuisances, sans pouvoir cependant se substituer aux détecteurs individuels pour l'évaluation exacte de l'exposition du personnel.

Les rejets d'effluents nécessitent une mesure aussi exacte que possible des activités rejetées et mettent le plus souvent en œuvre des mesures de laboratoire. Certaines mesures fixes peuvent intervenir pour déclencher une action tendant à réduire l'exposition du public.

Les caractéristiques principales des détecteurs sont signalées. Le choix des seuils d'alarme fait l'objet d'une distinction entre les détecteurs ayant une fonction de mesure et ceux ayant une fonction de détection d'évolution.

\begin{abstract}
An analysis of potential external exposure and atmospheric contamination in a PWR power plant shows out which monitoring devices are best suited. Fixed monitoring is required in some cases, mainly as evolution monitoring, however measurements by portable devices seem better for an assessment of hazards, yet they cannot supersede individual detectors for an accurate evaluation of workers' exposures.

Effluent activities must be measured as accurately as possible, which requires laboratories measurement. Some fixed monitoring can be planned so as to start an action in order to lower public exposure. The main features of the detectors are stated. When selecting alarm thresholds, detectors supplying measurements must be distinguished from those dedicated to evolution monitoring.
\end{abstract}

* Électricité de France, Direction de la production et du transport, Département de radioprotection, 6, rue Ampère, 93203 Saint-Denis. 


\section{INTRODUCTION}

Il paraît utile de réviser périodiquement, à la lumière de l'expérience acquise, la philosophie des moyens de mesure mis à la disposition de l'exploitant dans le but final de réduire les doses supportées par le personnel et le public.

L'action de réduction des doses passe tout d'abord par une évaluation des conditions d'exposition des personnes pendant le temps où elles sont soumises à l'action des rayonnements. Cette évaluation peut porter sur l'irradiation externe, pour limiter le temps d'exposition par exemple, ou sur la contamination atmosphérique ou surfacique, dans le but d'utiliser les moyens adéquats de protection respiratoire ou vestimentaire. Ceci est une des fonctions des mesures de radioprotection qui est généralement plus efficacement assurée par des matériels portatifs que par des matériels fixes.

Dans l'éventualité d'une évolution rapide des conditions d'exposition, on peut également demander à ces mesures de remplir une fonction d'alarme en vue de l'évacuation rapide des travailleurs, dès que l'évolution de la situation radiologique du milieu de travail révèle des circonstances anormales pouvant éventuellement préfigurer un accident.

Par ailleurs, les mesures de radioactivité constituent un moyen très sensible pour détecter les fuites des circuits actifs et peuvent entraîner des actions automatiques visant à la protection des personnes. Ces actions sont considérées comme des actions qui concourent à la sûreté dans la mesure où elles corrigent les défauts des matériels conçus pour confiner les sources de rayonnement à l'intérieur des circuits actifs.

\section{CLASSIFICATION DES MESURES DE RADIOPROTECTION}

L'analyse des conditions d'exposition des personnes au cours du fonctionnement d'une centrale nucléaire permet de subdiviser les mesures de radioprotection dans les catégories suivantes :

- mesures de protection directe du personnel visant à réduire son exposition immédiate;

- mesures de protection indirecte du personnel ayant pour but de prévenir une exposition ultérieure;

Ces mesures doivent remplir leur office aussi bien en exploitation normale que lors de circonstances accidentelles.

\section{DISPOSITIFS DE MESURES SERVANT A LA PROTECTION DU PERSONNEL}

Nous examinerons tout d'abord les mesures d'ambiance en dehors des périodes de travaux pour ne traiter ce cas particulier qu'en fin de paragraphe. 


\subsection{MESURES PÉRIODIQUES PAR APPAREILS PORTATIFS}

Il convient de préciser que, dans une centrale nucléaire, il n'y a pas de poste de travail fixe et permanent en zone contrôlée. Les accès n'ont lieu que pour des rondes, inspections ou manœuvres d'exploitation de courte durée, et travaux. Quelques locaux, cependant, font exception à cette règle (laboratoire, laverie, zone de transit); les risques d'exposition y sont limités et doivent être étudiés spécifiquement; ils ne concernent, de plus, qu'un personnel réduit et habilité.

La complexité de l'installation ne permet pas de disposer des mesures continues des différentes nuisances possibles dans chaque local. Aussi la zone contrôlée fait-elle l'objet de rondes périodiques au cours desquelles on mesure avec des appareils portatifs les valeurs de débit de dose (rayonnement $\gamma$, neutronique exceptionnellement) et de contamination régnant dans chaque local pour les afficher à l'entrée du local ou de la zone contrôlée. Ces informations suffisent pour estimer le temps de présence admissible et juger de la protection vestimentaire ou respiratoire à mettre en œuvre, pour autant que les différentes nuisances ne subissent pas d'évolution.

Les zones où l'exposition est la plus élevée (zone orange au-delà de $200 \mathrm{mrem} / \mathrm{h}$ et rouge au-delà de $10 \mathrm{rem} / \mathrm{h}$ ne sont accessibles qu'après évaluation des conditions d'exposition au moment et pendant l'accès, et délivrance d'une autorisation particulière visée par une personne compétente en matière de radioprotection.

Dans les lieux où les nuisances sont susceptibles de présenter des évolutions lentes ou brutales, il convient d'analyser les causes de ces évolutions pour déterminer les moyens appropriés pour leur détection.

\section{2. ÉVOLuTION DE L'IRRADIATION EXTERNE}

En dehors des périodes de travaux, analysées plus loin, une évolution de l'irradiation externe peut être le fait soit d'une source contenue dans un circuit, soit d'une contamination atmosphérique en gaz rares, contaminant prépondérant de l'atmosphère dans une centrale à eau pressurisée en service.

3.2.1. Dans le premier cas, source contenue dans un circuit, l'évolution peut être localisée en un certain nombre de points chauds, a priori bien connus : filtres, résines, réservoirs. Ces points sont toujours situés dans des locaux protégés, le plus souvent inaccessibles au personnel sauf en période de travaux. Il n'y a donc pas lieu d'y entretenir des mesures continues.

Si l'évolution est généralisée, suite à une rupture de gaine par exemple, elle est perceptible en n'importe quel point du circuit primaire. Pour la détecter, il suffit donc de faire une mesure continue du primaire qui est, en fait, une mesure de débit de dose, en un point représentatif du circuit primaire. On a choisi à cet effet un emplacement du circuit de contrôle volumétrique (RCV) où le temps de transfert de l'eau depuis sa traversée du cœur est suffisamment

voL. $14-\mathrm{N}^{\circ} 1$ 
long pour réduire l'activité de l'azote 16 à un niveau permettant de détecter des fluctuations dues à un apport de produits de corrosion ou de fission. Le but de cette mesure n'est pas de rendre compte de l'activité du primaire, qui est obtenue par mesure en laboratoire sur des échantillons périodiques d'eau primaire. Son but est de donner une indication sur l'évolution du débit de dose en provenance du circuit primaire et des circuits auxiliaires en liaison avec le primaire. C'est dans cet esprit que cette mesure est renvoyée dans le local d'échantillonnage du primaire pour prévenir l'agent qui procède au prélèvement de la nécessité éventuelle de précautions particulières lors du prélèvement (réduction du volume par exemple). Son renvoi également en salle de commande peut déclencher une ronde particulière dans les locaux concernés.

\subsubsection{Dans le deuxième cas, contamination atmosphérique en gaz rares, l'évolution peut être lente ou brutale.}

Si elle est lente, à la suite d'une fuite faible du circuit, on profite du fait que le contaminant principal est aisément véhiculé par les circuits de ventilation, sans rétention sur les surfaces ou les filtres, pour utiliser la mesure du gaz dans un circuit de ventilation comme moyen de détection de l'évolution de la fuite. A cet égard, selon que la fuite se situe dans le bâtiment réacteur ou dans tout autre local, on dispose de la mesure d'activité gaz sur l'air de l'enceinte ou de la mesure d'activité gaz à la cheminée.

La première mesure est très sensible lorsque l'air de l'enceinte n'est pas renouvelé; pour une activité du fluide primaire de $1 \mathrm{Ci} / \mathrm{m}^{3}$ en ${ }^{133} \mathrm{Xe}$, et pour une enceinte de $50000 \mathrm{~m}^{3}$ de volume, son seuil de mesure $\left(10^{-6} \mathrm{Ci} / \mathrm{m}^{3}\right)$ permet de déceler une fuite inférieure au litre d'eau primaire par heure.

La deuxième mesure est moins sensible en raison du débit important de l'air de ventilation; cependant, les lieux de fuite préférentiels se situent dans les locaux peu ou pas accessibles (circuits de traitement des effluents gazeux par exemple), de sorte que la fuite ne présente pas de risque immédiat pour le personnel. Lorsque l'on détecte une évolution de cette mesure, on procède à des mesures d'activité volumique des gaz et des aérosols par appareils mobiles sur les circuits de ventilation, où des tapes de prélèvement ont été ménagées à cet effet dans les zones de rayonnement faible, dans le but d'identifier, par mesures successives sur les différentes branches des circuits de ventilation, le local à l'origine de la contamination, et de repérer la fuite dans la mesure où elle n'a pas été détectée par les moyens normaux d'exploitation.

Une évolution brutale de la contamination atmosphérique en gaz rares susceptible de provoquer une évolution de l'irradiation externe, en dehors des périodes de travaux, ne peut avoir pour origine qu'une fuite importante du réservoir de stockage de gaz ou un accident de manutention de combustible, libérant les gaz rares contenus dans celui-ci. Le premier cas survenant dans des locaux non accessibles ne présente pas de risque pour le personnel et est détecté par la mesure à la cheminée. Le deuxième cas ne peut survenir que pendant les manutentions de combustible dans les piscines du réacteur ou de stockage, dans des périodes où la présence du personnel est indispensable. $L a$ mesure du débit de dose résultant de la contamination atmosphérique en gaz 
rares apparaît comme le moyen d'information le plus rapide sur ce type d'accident (une mesure de contamination atmosphérique, qui devrait être faite dans une gaine de ventilation pour être représentative, introduit un temps de réponse non négligeable).

\section{3. Évolution DE LA CONTAMINATION ATMOSPHÉRIQUE}

Compte tenu de la particularité déjà mentionnée que les contaminants principaux, pendant le fonctionnement d'une centrale PWR et en dehors des périodes de travaux, sont les gaz rares, on peut considérer que ce sujet a été traité dans le paragraphe précédent.

L'expérience montre que le rapport entre l'activité volumique des gaz rares et l'activité volumique des aérosols ou produits volatils est supérieur à 1000 , de sorte qu'un seuil de mesure en gaz rares de l'ordre de $10^{-6} \mathrm{Ci} / \mathrm{m}^{3}$ permet de répondre d'une contamination en aérosols ou produits volatils au plus égale à $10^{-9} \mathrm{Ci} / \mathrm{m}^{3}$.

\subsection{PÉRIOde de travauX}

En période de travaux mettant en cause l'étanchéité des circuits, l'irradiation externe et la contamination atmosphérique peuvent varier de manière quelconque selon les travaux effectués. Ceux-ci ayant lieu en n'importe quel point de l'installation, il n'est pas possible d'installer à poste fixe un matériel de mesure adapté à chaque emplacement de travail. En conséquence, on emploie un matériel mobile de chantier qui peut être aussi bien un détecteur de rayonnement $\gamma$ qu'un détecteur de contamination atmosphérique. Pour ce dernier point, les gaz rares ne constituant plus un traceur significatif, la mesure doit porter sur les aérosols, qui sont les contaminants essentiels produits par les travaux.

L'emploi du matériel mobile permet de placer le détecteur de rayonnement $\gamma$ directement dans la zone de travail et le détecteur d'aérosols au plus près de cette zone pour réduire la longueur de la tuyauterie de prélèvement, toujours préjudiciable à la représentativité du prélèvement. On a d'ailleurs développé un détecteur d'aérosols compensé du rayonnement $\gamma$ ambiant pour permettre cette proximité. Le détecteur continu de contamination atmosphérique en aérosols se justifie essentiellement si un travail est engagé sans qu'il ait été jugé nécessaire, par les estimations préalables, de munir les travailleurs d'une protection respiratoire. $\mathrm{Si}$, à l'inverse, un travail est engagé avec une protection respiratoire, un contrôle de contamination atmosphérique en cours de travail, par mesure sur un prélèvement sur filtre fixe de durée limitée avec aspirateur portatif, peut mettre en évidence que la protection est superflue et cause d'une augmentation du temps de travail, donc de la dose d'irradiation externe.

Pour les périodes de travaux de longue durée, au cours desquelles la contamination est jugée suffisamment faible pour ne pas nécessiter une protection respiratoire, il peut être nécessaire de procéder à un prélèvement continu des aérosols et des produits volatils pour vérifier, après mesure des échantillons en laboratoire, que la contamination intégrée par le personnel reste négligeable. 


\section{DISPOSITIFS DE MESURE \\ SERVANT A LA PROTECTION INDIRECTE DU PERSONNEL}

Les mesures de protection indirecte portent sur des lieux où ils convient de recueillir une information avant l'accès ou sur des points particuliers sur lesquels l'attention de l'exploitant doit être attirée pour éviter une exposition ultérieure excessive.

4.1. Dans la première catégorie - lieux où il est nécessaire de connaître les conditions avant l'accès - il a paru utile de ne retenir que deux emplacements : le bâtiment réacteur et le local de stockage des déchets actifs.

Dans le bâtiment réacteur d'accès peu fréquent en marche, il serait illogique de faire des mesures par appareils portatifs avant l'accès, ces mesures nécessitant elles-mêmes un accès. Aussi, dispose-t-on de la mesure d'activité volumique des gaz sur l'air de l'enceinte déjà citée, et d'un échantillonnage des aérosols et produits volatils sur le même prélèvement d'air. Des éléments perturbateurs, tels que les gaz rares naturels (radon) ou de fission et leurs aérosols de filiation, interdisent une mesure significative de l'activité volumique des aérosols et des produits volatils. L'évaluation de l'activité volumique est faite par mesure différée ( $\beta$ globale ou spectrométrie $\gamma$ ) en laboratoire des échantillons prélevés (filtre et piège). Les conditions d'ambiance sont ainsi suffisamment bien connues pour décider de la protection respiratoire adéquate. Ces prélèvements continus sont toujours disponibles pour mesure en laboratoire. En particulier, lorsqu'une évolution anormale est détectée par la mesure continue des gaz rares, il y a lieu de procéder au prélèvement des échantillons de poussières et produits volatils pour détermination, par spectrométrie $\gamma$, de l'activité volumique de ces contaminants dans l'enceinte.

Le local de stockage des déchets actifs non conditionnés fait l'objet d'apports discontinus susceptibles de créer des évolutions dans le débit de dose ambiant dont il convient de prévenir les personnes devant accéder à ce local ainsi que l'exploitant pour un enlèvement en temps utile des déchets en vue de leur conditionnement définitif. C'est à ces fonctions que répond la mesure continue du débit de dose dans le local de stockage.

4.2. Dans la deuxième catégorie - points où il est souhaitable de limiter l'activité pour éviter une exposition ultérieure excessive - on peut classer les mesures continues de débit de dose auprès des filtres ou déminéraliseurs où l'activité est susceptible d'évoluer de façon rapide, et les mesures de contamination des liquides séparés du fluide primaire par une simple barrière.

La mesure continue du débit de dose sur les filtres et déminéraliseurs $d u$ circuit primaire (RCV et TEP) a pour but d'arrêter l'utilisation du matériel concerné dès que l'activité contenue est susceptible d'entraîner :

- des doses excessives pour le personnel lors du remplacement du matériau, compte tenu des moyens de protection mis en œuvre à cette occasion; 
- ou, après conditionnement du déchet (filtre ou résines) dans un conteneur de béton, un débit de dose à l'extérieur du conteneur supérieur à celui admissible pour le transport.

Dans le cas de résines, un optimum économique est à rechercher entre le remplacement des résines et le nombre de conteneurs entre lesquels il est nécessaire de fractionner la charge de résines pour satisfaire aux conditions de transport. Pour aider au fractionnement des charges, il est nécessaire de placer une mesure continue de débit de dose sur le pot doseur de résines usées et de concentrats d'évaporateur avant mise en fut, ceci dans le but de limiter l'activité contenue dans chaque bloc de béton, donc le débit de dose au contact.

Les mesures de contamination des liquides séparés du fluide primaire par une simple barrière remplissent des fonctions de sûreté en tant que contrôle de l'étanchéité des barrières et de protection indirecte du personnel, dans la mesure où elles entraînent une action préventive (isolement du circuit par exemple) réduisant la contamination du circuit dans lequel fuit le circuit primaire.

A ce titre, le circuit secondaire eau-vapeur mérite une mention particulière parce que sa contamination peut être une cause de rejet d'effluents radioactifs dans l'environnement, de même qu'une cause d'irradiation du personnel. Deux voies de détection sont mises en œuvre : la mesure d'activité des incondensables extraits du condenseur, particulièrement sensible à une fuite brutale du générateur de vapeur créant un rejet notable de gaz dans la vapeur, et la mesure d'activité des purges de générateurs de vapeur, mieux adaptée à la détection d'une fuite faible non perceptible par la première voie, mais engendrant à terme plus ou moins long une contamination décelable dans l'eau des générateurs par concentration des produits radioactifs.

Une fonction analogue est remplie par les mesures d'activité de l'eau intermédiaire refroidissant les matériels du circuit primaire et de l'eau condensée à partir de la vapeur auxiliaire alimentant les dégazeurs et évaporateurs. De même, la mesure d'activité de l'eau sortant des échangeurs d'aspersion permet de détecter une fuite de ces échangeurs utilisés en période d'accident de rupture de tuyauterie primaire.

\section{CARACTÉRISTIQUES PRINCIPALES DES DÉTECTEURS ET SEUIL D'ALARME}

5.1. Des caractéristiques générales doivent être imposées à l'ensemble des détecteurs et vérifiées à la mise en service :

- étendue de mesure adaptée à la fonction;

- réponse satisfaisante, avec précision à définir, dans toute l'étendue de mesure souhaitée. Cette qualité doit être conservée quelles que soient les grandeurs d'influence telles que la température, la pression ou l'humidité du 
fluide, lorsque la mesure est faite sur un fluide, ou de l'ambiance. La plage de variation des grandeurs d'influence doit être précisée pour le fonctionnement normal et pour le cas d'accident. Les grandeurs d'influence peuvent comprendre également les vibrations dues au matériel environnant ou aux séismes, ou des éléments perturbateurs tels que des rayonnements parasites émis dans le fluide ou dans l'environnement;

- réponse toujours significative, soit du fait de la grandeur mesurée elle-même, soit du fait d'une source additionnelle, ceci pour pouvoir s'assurer en permanence du bon fonctionnement du détecteur et déclencher un signal en cas de défaut. Cette qualité doit être d'autant plus recherchée que le détecteur intervient dans la protection directe des personnes ou dans des circonstances accidentelles;

- représentativité du prélèvement en cas de mesure sur un fluide;

- constance de l'alimentation électrique obtenue soit par des sources extérieures fiables, soit par une source incorporée venant au secours de l'alimentation extérieure;

- signal d'alarme transmis aux points utiles (salle de commande ou poste local selon le cas) avec identification du type de défaut et rendu perceptible à tout le personnel exposé lorsqu'il s'agit de prévenir une exposition du personnel.

Par ailleurs, le contrôle de bon fonctionnement des alarmes doit être vérifié périodiquement par des moyens radioactifs. Il doit permettre de vérifier le déclenchement des signaux sonores et lumineux pour tous les cas où l'alarme s'accompagne de l'évacuation du personnel.

Enfin, le déclenchement du seuil d'alarme ne doit pas prêter à ambiguïté pour le personnel. Aussi avons-nous posé comme principe que le seuil d'alarme est unique et que son déclenchement doit provoquer une action : évacuation pour le personnel exposé, isolement du circuit ou manœuvre conservatoire, enquête et mesures appropriées pour déterminer la nature de l'incident (arrêt de ventilation ou passage sur piège à iode avec réduction de débit, par exemple).

\section{CONCLUSION}

L'analyse des possibilités d'irradiation dans une centrale nucléaire conduit à définir les matériels les mieux adaptés pour évaluer cette irradiation.

En raison de la complexité des locaux dans une centrale nucléaire et de l'absence de poste fixe et permanent de travail en zone contrôlée, l'estimation des nuisances est essentiellement faite par des apparails portatifs à l'occasion des rondes systématiques. Des détecteurs fixes doivent cependant déceler les évolutions de la contamination atmosphérique ou de l'irradiation externe susceptibles d'affecter le personnel.

Compte tenu de leur spécificité, les travaux doivent faire l'objet de mesures particulières par appareils portables. 
Quelques lieux d'accès peu fréquents, mais où l'information sur les nuisances est utile avant l'accès, font l'objet de mesures fixes. De même, certains points particuliers sont surveillés pour éviter une exposition ultérieure excessive du personnel.

Cependant, il n'est envisagé en aucun cas d'utiliser les appareils de mesure fixe, toujours peu représentatifs de l'exposition des personnes, pour évaluer les doses subies par le personnel. L'irradiation individuelle externe ne peut être déterminée que par des dosimètres individuels. Les protections mises en œuvre contre les contaminations ne nécessitent une évaluation de l'irradiation interne que dans des circonstances exceptionnelles, du domaine de la médecine du travail. 